\title{
Use of the Distance Transform for the Integration of Local Measurements: Principle and Application in the Field of Chemical Engineering.
}

\author{
Loïc Sorbier $^{1}$, Maxime Moreaud ${ }^{1}$, F. Bazer-Bachi ${ }^{1}$ and Virginie Moizan-Basle ${ }^{1}$ \\ ${ }^{1}$ IFP Energies nouvelles, Solaize, France
}

It is often necessary to convert local concentration measurements performed by Electron-Probe Micro Analysis or other spatial resolved techniques to global information at the sample scale. For example, for the analysis of heterogeneous catalyst, the concentration profile of uneven deposited elements in the grain have to be converted into a global information at the grain scale (total quantity of element [1], thickness of deposited element [2]). If the catalyst grain has a trivial shape (infinite plate and cylinder, sphere), simple analytical formula are amenable. Yet, non-trivial grain shapes cannot be handled so easily. Fortunately, some processes such as reaction-diffusion or imbibition lead to element distribution closely related to the distance to the surface of the sample. In that case, it is possible by a variable change to express the searched global quantity in function of the distance to the surface of the grain. The use of distance transform on image, allows to compute the desired global quantity with reasonable accuracy. Distance transform of a binary image is an image in which each pixel contains the minimal distance from this pixel to associated seeds, which are chosen in our case as the surface of the grain. It can be accurately and efficiently computed using queue algorithm and nearest neighbor transform [3]. In this work, we propose a general integration scheme allowing to compute global quantities at the grain scale from finite measurement points along a line and a picture of the section of the grain. We illustrate the application of this scheme on a simple model of reaction-diffusion chemical process.

The general scheme of integration is described in Figure 1 and consists in:

1) acquisition of a picture (optical or scanning electron microscopy) of the section of a grain

2) binarization of the picture and calculation of its distance transform

3) calculation of the density of points $\mathrm{d} n(y) / \mathrm{d} y$ in the grain at a distance $y$ from the surface

4) calculation of $y$ versus the distance to the start of a line profile $x$

5 ) acquisition of measurement along the line profile and integration of the desired quantity

The example of application originates from a simple model of metal deposition during hydroprocessing of oil residues [4]. We assume a first order kinetic and neglect both the transitional regime and the plugging of the pores of the catalyst. With these rough assumptions, the global concentration $C$ of deposited metal and the mean depth of metal deposit $L$ is given by simple analytical formula for the trivial shapes. These quantities are explicitly dependent of a well-known kinetic parameter in chemical engineering: the Thiele modulus $\phi[5]$.

First, we compare the result obtained with the proposed integration scheme with the theoretical results on the cylinder for a large range of Thiele modulus. We particularly study the influence of the line profile parameters (number of points, constant or variable step) on the accuracy. The proposed strategy allows to obtain a reasonable accuracy ( $<1 \%$ relative) for moderate values of the Thiele modulus where the concentration profile can be reasonably sampled. In a second step, we show that the approach still gives reasonable accuracy on multilobed grain shapes. Thus, this work propose an original method of integration of local measurement for non-trivial shapes of samples and a parametric study of the accuracy obtained by the method. 


\section{References:}

[1] L. Sorbier in "Catalysis by Transition Metal Sulphides", ed. H. Toulhoat and P. Raybaud, p.407.

[2] L. Sorbier et al, IOP Conf. Ser.: Mater. Sci. Eng. 32 (2012) 012023.

[3] L. Ikonen and P. Toivanen, Proceedings of the $7^{\text {th }}$ international conference on Advanced Concepts for Intelligent Vision Systems (2005) p.308.

[4] M. Roy-Auberger et al in "Catalysis by Transition Metal Sulphides", ed. H. Toulhoat and P. Raybaud, p.690.

[5] K. Bischoff, AIChE Journal 11 (1965), p.351.

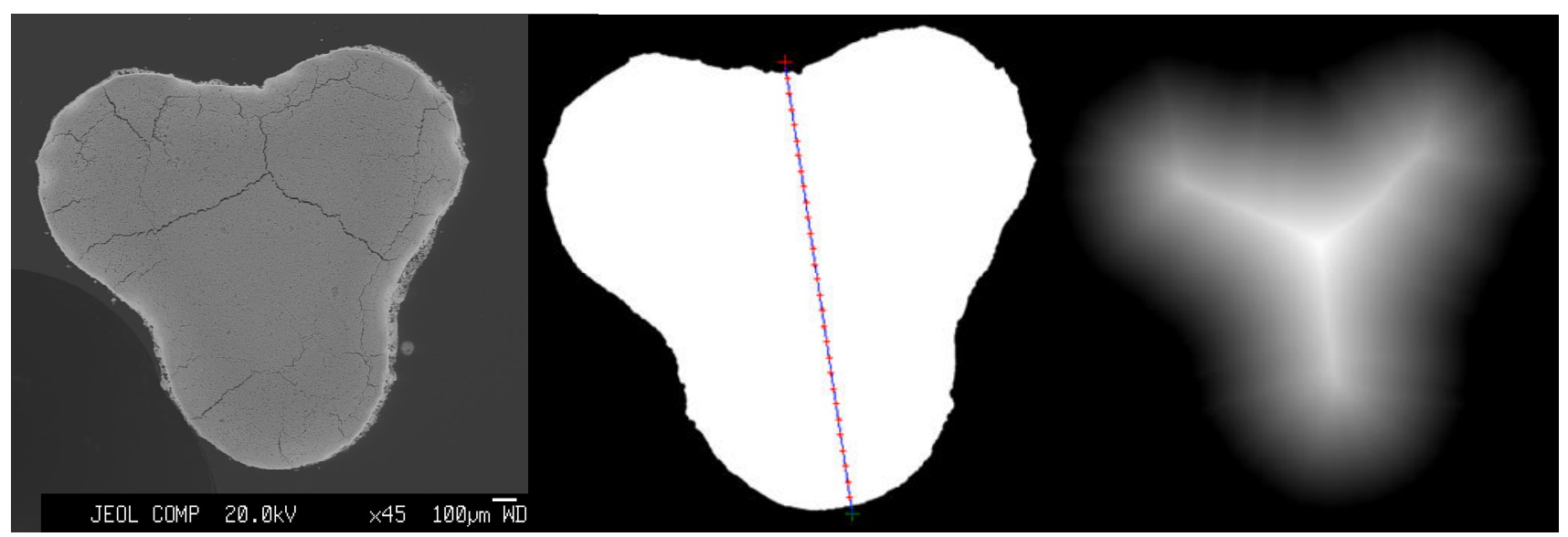

(a)

(b)

(c)

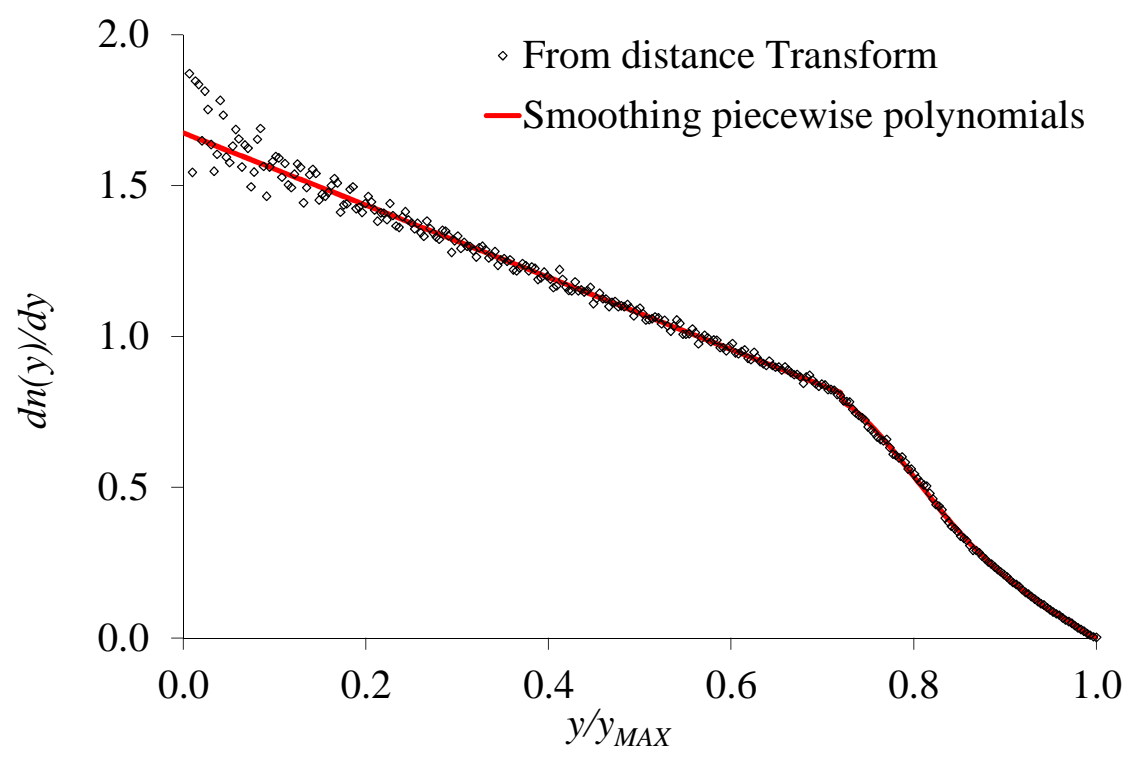

(d)

Figure 1. General scheme to integrate profile line measurements. (a) Backscattered electrons image of the section of a trilobe (b) Segmented image and line profile points in red (c) Associated distance map (d) Density of points at a distance $y$ to the surface of the grain as measured by distance transform and smoothing piecewise polynomials. 